\title{
Un premier bilan des effets psychiques et sociaux de l'accident de Tchernobyl*
}

\author{
G. HERIARD DUBREUIL**
}

(Manuscrit reçu le 11 février 1994)

RÉSUMÉ Cet article présente la synthèse d'une série d'enquêtes réalisées en Ukraine en 1992 et 1993 sur les conséquences psychiques et sociales de l'accident de Tchernobyl, dans le cadre du "Programme d'évaluation des conséquences de l'accident nucléaire de Tchernobyl" de la Commission des Communautés Européennes. Les principaux résultats confirment la puissance de la dynamique post-accidentelle de cet accident, après plus de sept ans. Près de 3 millions de personnes sont directement concernées dans leur vie quotidienne par le dispositif de gestion post-accidentelle qui est à l'origine de nombreux effets pervers aux plans social et psychique. Au plan économique, le financement du dispositif de gestion post-accidentelle absorbe chaque année près de $1 / 6$ du budget de l'état ukrainien. Au plan politique, Tchernobyl est encore un enjeu majeur pour les différents acteurs du processus de transition institutionnel engagé depuis la disparition du système soviétique. L'article montre la complexité systémique de la situation rencontrée sur le terrain et présente les multiples facteurs explicatifs (physiques, sanitaires, politiques, culturels, historiques) qui sont à l'origine de la dynamique post-accidentelle. Une modélisation systémique des interactions entre ces facteurs est proposée. Elle permet de mieux cerner la contribution des différentes étapes accidentelles et post-accidentelles dans le processus qui a conduit à la situation actuelle. Elle montre notamment les liens étroits entre les différentes étapes de l'accident et la nécessité de prendre en compte, dès le début d'un accident, les conséquences à moyen et long terme qui découlent de la gestion accidentelle.

ABSTRACT A synthesis has been made of a series of surveys carried out in Ukraine in 1992 and 1993 on the psychic and social consequences of the Chernobyl accident, within the framework of the "Evaluation programme of the consequences of the Chernobyl nuclear accident" of the Commission of the European communities. The main results demonstrate the strength of the post-accident dynamics of the accident, more than 7 years later. Some 3 millions people were directly affected in their everyday life by the post-accident management which resulted in many perverse effects on the social and psychic levels. Economically, each year, financing of the post-accident management system requires nearly 1/6 of the Ukraine budget. Politically speaking, Chernobyl is still a major stake for the various actors of the institutionnal transition process underway since the disappearance of the soviet system. The article shows the systemic complexity of the local situation and the many explanatory factors (physical, sanitary, political, cultural, historical) at the origin of the post-accident dynamics. A systemic modelling of

Les travaux dont cet article présente la synthèse ont été réalisés avec le soutien de la Commission des communautés européennes dans le cadre de ses actions de recherche en radioprotection.

** Mutadis Consultants, 14, rue de Belzunce, 75010 Paris. 
the interactions between these factors is presented. It makes it possible to better define the contribution of both accident and post-accident stages to the process that has led to the present situation. It shows out the close connections between the different accident stages and the need, from the very beginning of an accident, to take into account the mid- and long-term consequences arising from the accident management.

\section{Introduction}

L'analyse de l'impact socio-économique de l'accident au plan national, sept ans après celui-ci, confirme la puissance de la dynamique post-accidentelle de Tchernobyl en Ukraine. Au plan administratif, ce sont près de 3 millions de personnes : anciens participants à la gestion de l'accident (liquidateurs), habitants des territoires contaminés, personnes relogées hors des territoires contaminés, qui sont directement concernées dans leur vie quotidienne par les modalités du dispositif de gestion post-accidentelle mis en place par la loi ukrainienne du 16 avril 1991. Celui-ci comprend, notamment, un régime de compensation à travers un système complexe de primes et d'avantages en nature qui est à l'origine d'effets indésirables sur ces populations, au plan psychique et au plan social. Au plan économique, le financement du dispositif de gestion post-accidentelle, dans lequel l'Ukraine rencontre de graves difficultés, absorbe chaque année près de $1 / 6$ du budget de l'état ukrainien. Cette somme est financée par une taxe de $12 \%$ sur les salaires et gérée par une administration spécifique, le "ministère Tchernobyl". Plus de $36000 \mathrm{~km}^{2}$, contaminés à plus de $37.10^{9} \mathrm{~Bq} / \mathrm{km}^{2}$, sont soumis à des restrictions de consommation alimentaire et de développement économique. Au plan politique enfin, Tchernobyl reste un enjeu majeur pour les différents acteurs du processus de transition institutionnelle engagé depuis la disparition du système soviétique.

Cet article présente une première synthèse des enseignements que l'on peut tirer d'une série d'enquêtes réalisées en Ukraine en 1992 et 1993 [5] sur les conséquences psychiques et sociales de l'accident de Tchernobyl, dans le cadre du "Programme d'évaluation des conséquences de l'accident nucléaire de Tchernobyl" (Joint study project 2) qui est développé par la Commission des Communautés Européennes (DG XII) [1, 4-5]. Dans une première partie, nous exposons les principaux résultats de ces enquêtes. Dans une seconde partie, nous présentons un certain nombre de facteurs explicatifs qui se sont dégagés de ces enquêtes ainsi qu'une proposition de modélisation systémique des interactions entre ces facteurs. Ces éléments permettent de dégager une première interprétation de situation post-accidentelle. 


\section{Méthodologie}

L'objectif de ces enquêtes était de réaliser une évaluation qualitative des détriments psychiques et sociaux subis par la population des territoires contaminés par l'accident de Tchernobyl ainsi que par les populations évacuées puis relogées après cet accident. Sur un plan méthodologique, l'évaluation a été réalisée à partir. d'un travail d'interprétation du matériel d'enquête recueilli sur le terrain en 1992 et 1993. La principale difficulté de ce travail résidait dans le fait que les personnes interrogées sont sujettes à des résistances individuelles ou collectives, conscientes ou non conscientes. Ces résistances, comme l'existence de non-dits, créent une opacité du discours vis-à-vis de la question étudiée qui rend difficile une interprétation directe. Il était également nécessaire de prendre en compte les multiples distorsions induites par les discours politiques et médiatiques depuis l'accident (depuis plus de sept ans). Différentes méthodes ont donc été mises en œuvre pour déceler, de façon indirecte, les détriments psychiques subis par la population à travers un travail d'interprétation du matériel recueilli sur le terrain.

Le recensement des déchirures du tissu psychique et social a été réalisé à différents niveaux. A travers l'analyse des représentations de l'accident et des contre-mesures, cette recherche s'est attachée particulièrement à déceler les formations réactionnelles, les défenses élaborées aux plans individuel et collectif pour parer à l'intensité des traumatismes (remaniement des représentations, déni de la réalité, oubli, retrait dépressif, mesures contraphobiques, fuite dans le passé ou dans la tradition, etc). De façon générale, l'étude a permis de mettre en évidence les effets paradoxaux ou contre-productifs des mesures accidentelles et post-accidentelles, engendrés de proche en proche et imperceptiblement par les mécanismes habituels. L'étude a également pris en compte les effets de l'accident sur le lien social (désagrégation, rupture des socialités primaires et secondaires), notamment au sein des populations déplacées.

Parallèlement, une étude détaillée du dispositif administratif et juridique ukrainien de gestion post-accidentelle (loi du 16 avril 1991) a été réalisée pour évaluer les éventuels effets paradoxaux induits, psychiques ou sociaux, des contre-mesures post-accidentelles.

Deux enquêtes sur le terrain ont été réalisées en décembre 1992 et avril 1993. Les investigations sur le terrain ont été menées de façon systématique, sur la base d'une sélection aléatoire, auprès des populations relogées ainsi que dans les différentes zones de contamination, soit (pour ${ }^{137} \mathrm{Cs}$ ) de 1 à $5 \mathrm{Ci} / \mathrm{km}^{2}$ $\left(37-185.10^{9} \mathrm{~Bq} / \mathrm{km}^{2}\right)$, de 5 à $15 \mathrm{Ci} / \mathrm{km}^{2}\left(185-555.10^{9} \mathrm{~Bq} / \mathrm{km}^{2}\right)$, plus de $15 \mathrm{Ci} / \mathrm{km}^{2}$ (plus de $555.10^{9} \mathrm{~Bq} / \mathrm{km}^{2}$ ), dans la zone interdite, ainsi que dans une zone de contrôle non contaminée (plus de $100 \mathrm{~km}$ à l'est de Kiev); 135 entretiens qualitatifs d'une durée de 30 à 90 min ont été réalisés sur la base d'un questionnaire semi-directif. Une collaboration avec plusieurs centres scolaires des territoires contaminés ainsi que des localités de relogement a permis de rassembler environ 150 rédactions libres (sur le thème de Tchernobyl) d'enfants âgés de 7 à 
14 ans. Des entretiens avec des adhérents de différentes associations liées à l'accident comme "Soyouz Tchernobyl" ou "Maman 86" ont également été réalisés.

Après la phase de collecte, les données recueillies ont été transcrites, traduites puis analysées par un groupe interdisciplinaire d'universitaires et de consultants (sémiologie, communication, psychologie, psychanalyse, sociologie). L'analyse de contenu a été conduite selon les méthodes classiques complétées par les méthodes suivantes:

- repérage des complexes de mots et des ensembles sémantiques répétitifs ;

- repérage des liaisons fréquentes et des univers sémantiques.

Ces méthodes complémentaires visent l'identification des non-dits, métaphores, formes allégoriques des signifiants sensibles et particulièrement chargés (accident nucléaire, irradiation, doses, contamination, zone, contre-mesure, liquidateur, issus du vocabulaire technique, médico-sanitaire et organisationnel) ainsi que du halo sémantique entourant ces signifiants.

\section{Principaux résultats}

\subsection{Un climat d'anxiété généralisé}

Ces investigations ont montré, sur l'ensemble des zones, un niveau de perturbation psychique préoccupant, qui est clairement associé aux conséquences de cet accident. Un climat d'anxiété généralisé est observé dans les différents groupes de population étudiés (relogés, habitants des territoires contaminés, participants à la liquidation de l'accidènt). Cette anxiété concerne les effets de l'accident de Tchernobyl sur la santé des personnes interrogées et sur celle de leurs proches. Elle s'exprime tout particulièrement à propos de la santé des enfants. L'expression de cette inquiétude est assortie, dans les interviews, de multiples références à des effets somatiques divers constatés par la population et systématiquement attribués par celle-ci à l'accident de Tchernobyl, parmi lesquels des références constantes à des troubles de la thyroïde et la mention de fréquentes "morts subites" sans causes apparentes, particulièrement chez les hommes adultes.

Ce constat est particulièrement aigu dans les populations relogées au sein desquelles il est très rare de déceler les symptômes d'un véritable processus de réparation psychique et sociale des suites du traumatisme provoqué par leur évacuation définitive. La possibilité d'un oubli et d'un retour à la normalité semble généralement condamnée par un sentiment de perte irréparable qui conduit, le plus souvent, les personnes interrogées à parler de leur vie sous la forme d'un "avant" et d'un "après" l'accident de Tchernobyl. On note, dans les interviews, l'usage multiple de termes introduisant des références historiques ou symboliques à une situation de guerre et d'après-guerre : héros, décorations, compensations, exodes, déportations, invalides, priorités, cartes, réductions, etc. Cette "atmosphère de guerre" est d'ailleurs caractéristique de l'ensemble du pro- 
cessus de prise en charge de l'accident par le système soviétique (intervention massive de l'armée, mobilisation des réservistes, référence aux comportements héroïques [6] face au "monstre-accident", appel au civisme), puis par l'Ukraine (création de systèmes de pensions, de cartes d'anciens liquidateurs, etc).

La contamination de l'environnement est une préoccupation centrale pour l'ensemble des personnes interrogées. Celles-ci expriment leur inquiétude quant aux effets de cette contamination sur leur santé et celle de leurs proches. Cette peur s'accompagne d'un climat de défiance vis-à-vis du discours des pouvoirs publics ainsi que des autorités scientifiques et médicales. L'analyse des interviews montre que cette peur ne s'apparente pas à un syndrome clinique de phobie, dans son acception psychiatrique ou psychanalytique. Les personnes interrogées expriment leur crainte et leur anxiété, mais ce n'est jamais sous la forme d'une peur irraisonnée et d'une angoisse immaîtrisable. Ces peurs sont toujours étayées par un discours rationnel construit sur les observations réalisées par ces personnes. Les entretiens menés avec des enfants phobiques hospitalisés (Centre ukrainien de médecine radiologique) ont montré qu'il ne s'agissait pas de phobies rapportées à l'accident et les syndromes observés n'excèdent pas le cadre et le contenu classique des phobies infantiles.

La notion confuse de radiophobie, qui a été avancée très tôt après l'accident pour tenter d'expliquer les réactions de la population, ne semble avoir aucune pertinence clinique. Elle a d'ailleurs été progressivement abandonnée par les autorités médicales depuis 1990. A ce concept s'est substitué celui de "stress aigu et chronique" qui est actuellement utilisé par les médecins pour décrire les symptômes rencontrés dans la population des territoires contaminés comme dans les populations relogées. Ce concept de stress est historiquement dérivé de celui de névrose traumatique [2]; il concerne non seulement le champ proprement psychique mais aussi le registre biochimique et neurophysiologique. Les médecins présentent, donc, une conception élaborée du stress qui se démarque de son utilisation populaire et médiatique. Ils rapportent ce stress à une situation continue de désadaptation biologique, psychique et sociale qui exige de l'organisme, du système nerveux et de l'espace psychique un état d'alerte, de tension et de dépense d'énergie permanents entraînant des effets pathologiques tant organiques que psychiques.

Les personnes interviewées expriment leur profonde inquiétude quant à l'avenir. Les effets futurs de l'accident semblent être particulièrement redoutés, plus encore que les effets passés. Ce sentiment alimente une vision pessimiste et fataliste de l'avenir, particulièrement frappante chez les enfants de relogés. La conviction que la situation ne peut qu'empirer semble renforcer la passivité des personnes interrogées.

Ces observations rejoignent celles du Robens Institute [1] qui met en évidence, chez ces personnes, un sentiment aigu d'absence de maîtrise personnelle de leurs conditions d'existence et montre, en outre, que ce sentiment de nonmaîtrise est clairement corrélé au niveau élevé de détresse psychologique décelé par les tests dans ces populations. 
Les enquêtes montrent également que les concepts de gestion post-accidentelle sur lesquels repose la loi d'avril 1991 ont un effet aggravant sur le traumatisme psychique et social des populations concernées : relogés, habitants des territoires contaminés, liquidateurs (Fig. 1). Le système administratif de compensation mis en place par la loi ukrainienne de 1991 exerce une influence déterminante sur la vie quotidienne des personnes interrogées. L'étude montre que ces modalités d'évaluation des détriments (passés et futurs) causés par l'accident de Tchernobyl et les modalités de compensation prévues par ce système contribuent à freiner le retour à la normale des conditions de vie de ces personnes. Le principe de compensation des détriments n'est pas, en lui-même, à l'origine d'effets négatifs, mais ce sont ses modalités d'application qui ont des effets pervers. On peut même penser que le principe de compensation, s'il était correctement mis en œuvre, devrait même avoir des vertus thérapeutiques au plan psychique et au plan social.

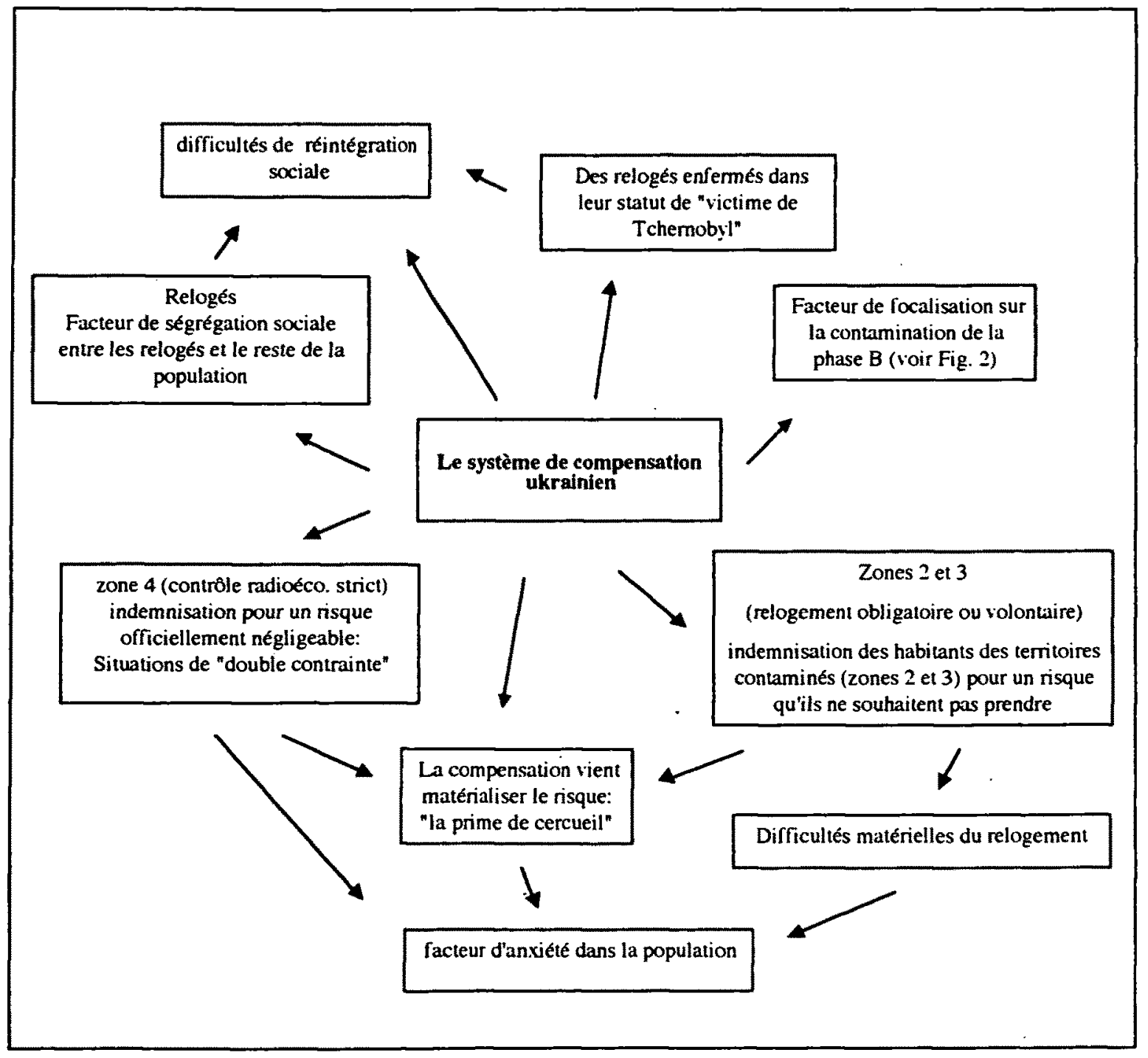

Fig. 1 - Les effets pervers du système de compensation ukrainien (loi du 16 avril 1991). Perverse effects of the Ukrainian compensation system (law of April 16, 1992). 
Ce système de compensation contribue notamment à enfermer les relogés dans un statut passif de "Tchernobylien" qui marque quotidiennement leur vie personnelle et leur vie sociale (l'accident vit à travers le système de compensation). Ce statut est également un facteur de ségrégation sociale, car il suscite la jalousie des populations autochtones à l'encontre des relogés et contribue donc à freiner leur réintégration sociale.

Les enquêtes montrent que les concepts mêmes sur lesquels repose la gestion des territoires contaminés (relogement volontaire, contrôle radioécologique strict, compensations financières communément appelées "prime de cercueil" par la population) sont à l'origine de situations de grave détresse psychologique au sein des populations concernées. L'étude des mécanismes de compensation et l'analyse des interviews montrent que les bénéficiaires du système de compensation sont placés dans une situation paradoxale qui s'apparente à une situation de double contrainte [3]. Elle génère, chez ces personnes, un niveau de stress élevé et les conduit à des attitudes de retrait, de désespoir et d'apathie.

\subsection{Le cas particulier des "samossiols"}

Un petit nombre de personnes (800 environ), généralement âgées, qui avaient été évacuées lors de l'accident puis relogées, ont enfreint les interdictions de résidence concernant la zone interdite (ancienne zone des $30 \mathrm{~km}$ autour du réacteur accidenté) et sont revenues habiter de leur propre chef dans leurs anciennes maisons. Ayant enfreint la loi, ces personnes, qui sont désignées sous le nom de "samossiols" (ce qui veut dire : "celui qui s'est institué de lui même") ne bénéficient d'aucun statut administratif et d'aucune compensation matérielle ou financière liée à leur résidence sur un territoire contaminé. Les enquêtes montrent que malgré une situation matérielle très difficile, malgré l'absence de famille et l'isolement social, malgré l'absence de perspectives futures, la santé psychique de ces personnes semble paradoxalement meilleure, en moyenne, que celle des habitants des territoires contaminés interwievés à l'extérieur de la zone interdite. En particulier, la contamination de leur environnement ne semble pas être un réel sujet de préoccupation chez ces personnes qui n'attribuent généralement pas les maladies dont elles peuvent souffrir à l'accident de Tchernobyl.

Notons, cependant, que ces observations ne concernent que la dimension psychique du traumatisme subi par ces personnes et ne préjugent en aucun cas des effets somatiques de l'accident sur ces personnes, dont l'évaluation précise reste à conduire.

Cette observation conduit, néanmoins, à penser que l'attitude mentale volontaire qui a conduit ces personnes à quitter leur lieu de relogement, de leur propre chef, pour se réinstaller chez elles, malgré les interdictions (et ceci en bravant les autorités), constitue un des facteurs explicatifs de l'état de moindre détresse psychologique de ces personnes. De même, le fait de ne pas bénéficier du système de compensation a pu, d'une certaine manière, protéger ces personnes des effets pervers de celui-ci en limitant leur dépendance psy- 
chologique. L'idée qui est développée ici n'est pas qu'une assistance matérielle ou financière est néfaste en elle-même, mais plutôt qu'elle doit être conduite sans créer chez ses bénéficiaires une attitude de dépendance et de démission.

\subsection{Multiplicité des facteurs explicatifs}

Les interviews révèlent la complexité de la situation constatée sur le terrain. De multiples facteurs explicatifs (historiques, physiques, sanitaires, politiques, culturels, etc) semblent être à l'origine de la situation rencontrée aujourd'hui en Ukraine. Tous ces facteurs contribuent, à des degrés variables, à la compréhension de cette situation, mais aucun d'entre eux ne peut suffire à l'expliquer. Ces facteurs sont eux-mêmes en interaction, ce qui nous conduit à parler de complexité systémique dans le sens qui a notamment été développé par différentes équipes de recherche en France et aux USA [7, 10].

En particulier, il est nécessaire de prendre en compte le fait que cette situation résulte de l'enchaînement des différentes situations antérieures qui se sont succédés depuis l'accident, qui semblent toutes, bien qu'à des degrés divers, contribuer au résultat actuel. Ainsi, de nombreux facteurs historiques semblent être à l'origine de la situation post-accidentelle. Une reconstitution des différentes phases de la gestion accidentelle et post-accidentelle et l'analyse de leurs caractéristiques et de leur impact sur la population sont donc une étape nécessaire dans une démarche de compréhension de cette situation. Ce travail de reconstitution étant réalisé, il est possible, à partir des différents facteurs identifiés, de tenter une interprétation des résultats de ces enquêtes et de proposer une modélisation des différentes interactions à l'origine de cette complexité.

\section{Tentative de modélisation}

Pour dégager les différents facteurs explicatifs, nous proposons de distinguer, d'une part, les effets physiques de l'accident à court, moyen et long termes et, d'autre part, les conséquences des modalités de la gestion accidentelle et post-accidentelle (Fig. 2).

\subsection{Des effets physiques différenciés}

Les effets radioinduits de l'accident ne sont pas tous de même nature dans le temps et nous proposons de distinguer les effets de la phase accidentelle de ceux de la phase post-accidentelle où subsiste une contamination résiduelle (celle qui est actuellement constatée), avec une partie seulement des radionucléides présents juste après l'accident. Par convention, nous appellerons phase A la période qui s'écoule du début de l'accident jusqu'à la fin de la construction du sarcophage (d'avril à novembre 1986) et nous appellerons phase $B$ la période suivante.

Les effets de la phase A résultent d'un environnement physique aujourd'hui disparu. Certains effets sont apparus très rapidement (effets déterministes), mais 
des effets différés sont, semble-t-il, en train d'apparaître aujourd'hui, ainsi que le montrent les résultats des études récentes menées par différentes équipes (OMS, $\mathrm{CEE}$, experts de la santé publique ukrainiens et biélorusses) sur les conséquences médicales de Tchernobyl $[9,11]$, qui s'accordent à constater une augmentation significative des taux de cancer de la thyroïde en Ukraine et en Biélorussie.

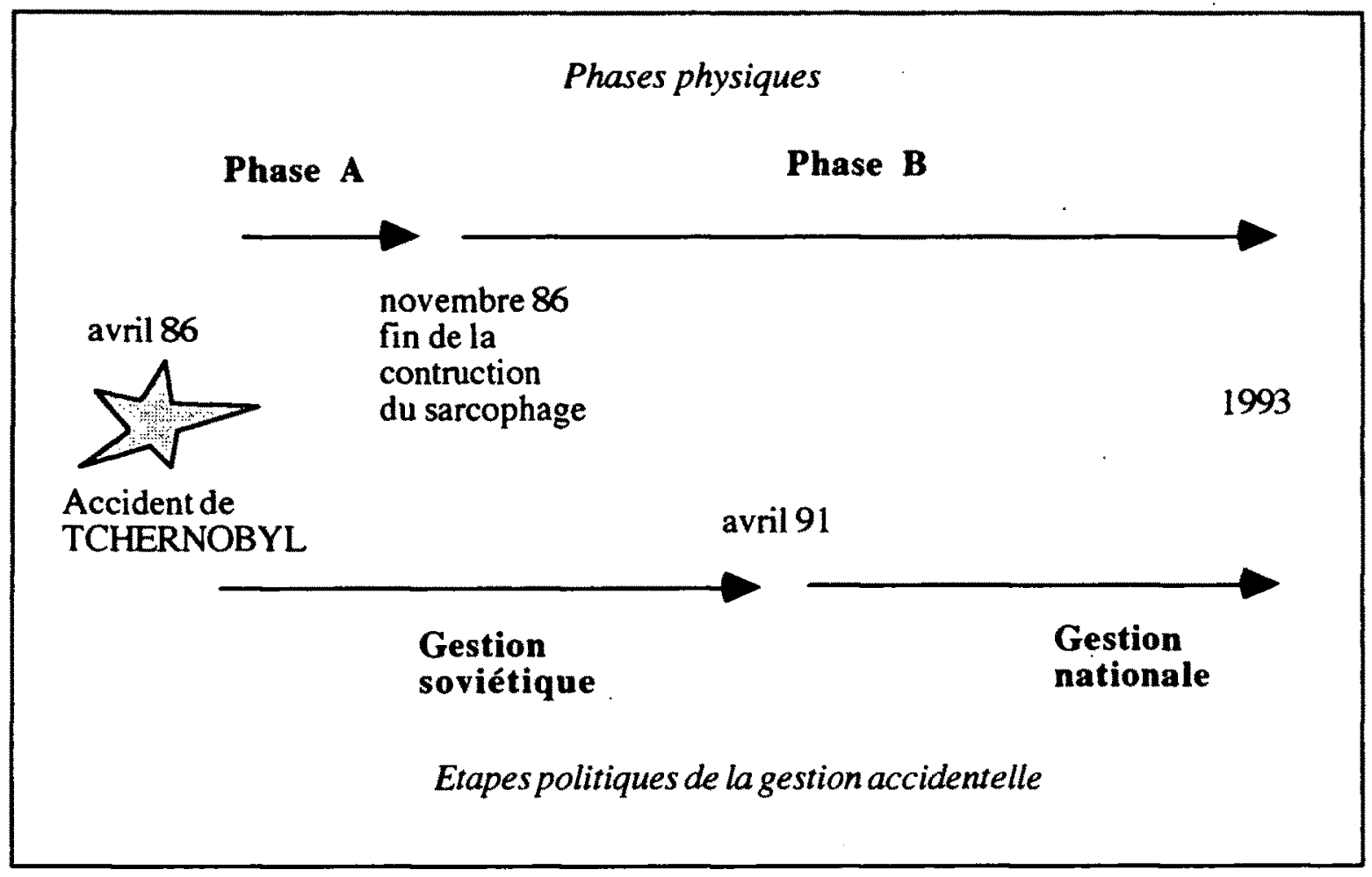

Fig. 2 - Les étapes physiques et politiques de l'accident.

Physical and political stages of the accident.

Les effets potentiels de la phase B qui pourraient résulter de la contamination résiduelle relèvent de la catégorie des effets stochastiques. Ils restent, pour le moment, difficilement repérables dans la population.

Notons que les problématiques associées à ces deux phases sont très différentes, en particulier dans leur durée (6 mois pour la phase A qui est terminée, bientôt plus de 7 ans pour la phase $B$ dont la durée est fonction de celle des radionucléides restants). La phase $A$, d'une certaine manière, relève d'une problématique du passé, même si des effets différés peuvent encore apparaître. Les effets de la phase A résultent d'une atteinte passée du capital de santé de la population. A l'inverse, la problématique associée à la phase $\mathrm{B}$ est actuelle et future, dans la mesure où ses effets résultent d'une cause physique qui ne disparaîtra que très progressivement.

\subsection{Les différentes modalités de gestion}

Sur un plan historique, il est également nécessaire de distinguer la "gestion soviétique" de l'accident (de 1986 à 1990), des "gestions nationales" (ukrai- 
nienne, biélorusse et russe) qui lui ont succédé (à partir de 1991), caractérisées par des pratiques différentes avec des conséquences différentes.

Les entretiens font ressortir les caractéristiques spécifiques de ces deux étapes qui laissent une empreinte profonde sur la représentation de l'accident et de ses conséquences par la population. C'est, en effet, à partir de la représentation mentale qui s'est construite au fil de leur expérience depuis l'accident ainsi que des informations dont ces personnes ont disposé au long de ce cheminement que celles-ci dégagent leur propre compréhension de la situation.

\section{a) La gestion soviétique}

Concernant la première étape dite de "gestion soviétique", les enquêtes font ressortir trois caractéristiques majeures qui semblent être à l'origine d'effets directs ou indirects au plan somatique, comme au plan psychique.

- La première caractéristique concerne l'absence de protection des populations durant la phase A (à l'exception des mesures d'évacuation qui ont souvent été tardives, notamment pour les populations rurales) et en particulier l'absence de distribution d'iode, à de très rares exceptions près. Ces observations faites par la population, si elles sont confirmées, pourraient expliquer l'apparition de pathologies de la thyroïde aujourd'hui constatées par les médecins. De même, ne peut-on établir un lien entre ces observations et la dégradation générale de l'état de santé rapportée par la population? Pour la bonne compréhension des conséquences psychiques et sociales de l'accident, cette question n'est pas superflue. L'évaluation de la réalité des effets somatiques rapportés par la population et leur lien éventuel avec l'accident sont déterminants. La compréhension de la situation rencontrée différera, en effet, radicalement selon que ces effets somatiques sont réels ou imaginaires et selon qu'ils sont directement et physiquement liés à l'accident ou non. On distinguera ici clairement les effets somatiques directs (immédiats ou différés) d'une exposition ou d'une contamination durant la phase $\mathrm{A}$, d'éventuels effets indirects psychosomatiques. Les résultats des investigations médicales citées plus haut conduisent à formuler l'hypothèse que la population est bien confrontée à l'émergence d'effets somatiques réels d'une part, et que ces effets, au moins pour une partie d'entre eux (pathologies de la thyroïde, en particulier), sont des effets différés de la phase A. Il faut, probablement, prendre en compte, également différents facteurs susceptibles d'aggraver un mauvais état sanitaire, comme le haut niveau de détresse psychologique qui peut être à l'origine d'effets indirects psychosomatiques, mais aussi comme la dégradation du climat économique dans les pays de la CEI (baisse de la qualité de la nourriture, pénurie de médicaments). Il faut également noter que le développement du suivi médical et de l'épidémiologie sur certaines catégories de population depuis l'accident a pu contribuer à révéler un mauvais état de santé publique antérieur à l'accident.

- La seconde caractéristique de la première étape de "gestion soviétique" réside dans l'usage de la censure et du secret par les autorités soviétiques durant la phase A, puis durant la phase B jusqu'en 1990 approximativement. 
Les interviews rapportent que les déclarations officielles durant la phase A sont systématiquement euphémiques. Les enquêtes montrent que les informations susceptibles de révéler à la population l'ampleur de l'accident et de ses conséquences ont été mises au secret. En particulier, les mesures de doses reçues par la population et par les participants à la liquidation de l'accident n'ont pas été communiquées à la population (si elles ont été réalisées ?). Cette attitude des autorités semble avoir eu des conséquences déterminantes au plan psychique. Elle s'est traduite par une absence de représentation et de symbolisation de cette phase A par la population qui a conduit, elle-même, à une confusion des effets des différentes phases (A et $\mathrm{B}$ ) de l'accident et, par conséquent, à une focalisation sur la phase $B$. Cette observation est l'un des éléments clés de la compréhension de la situation post-accidentelle de Tchernobyl.

- La troisième caractéristique de cette première phase est le nombre considérable des intervenants dans la gestion accidentelle. Les estimations ne sont pas toutes concordantes, mais on évalue généralement à 600000 personnes pour l'ensemble de l'ancienne Union Soviétique, le nombre de ces liquidateurs (certains organismes, comme l'OMS [8], font état de 800000 intervenants engagés à divers titres dans les opérations d'urgence). L'intervention de ces personnes a été suivie de leur dissémination dans la population, sans qu'une évaluation sérieuse des risques sur leur santé ne semble avoir été réalisée (non reconstitution des doses reçues). Ce nombre peut s'expliquer par l'ampleur des travaux à réaliser après l'accident mais aussi, compte tenu des niveaux d'exposition, par la nécessité de répartir le risque radiologique sur une très large population. Il faut également noter que la plupart de ces liquidateurs sont des civils (hommes et femmes résidant dans un rayon de plusieurs dizaines de kilomètres autour de la centrale) ou des militaires qui, en grande majorité, étaient des réservistes mobilisés dans toute l'URSS, puis démobilisés et renvoyés chez eux sans qu'un suivi médical individuel n'ait été mis en place. L'incertitude qui règne sur le risque auquel sont exposées ces personnes, sur les doses reçues, sur l'état sanitaire de ce groupe, nous semble également être un facteur de confusion pour la population qui semble très préoccupée par l'état de santé des liquidateurs, sans toutefois pouvoir se représenter clairement le risque encouru par ceux-ci (cette incertitude est fortement ressentie par les liquidateurs euxmêmes). Notons qu'étant donné l'importance de ce groupe (près de 200000 personnes en Ukraine), une éventuelle dégradation de son état sanitaire conjuguée à une incertitude sur l'origine de cette dégradation (pas de distinction claire entre phase A et phase B) peut constituer un puissant facteur de confusion et d'inquiétude dans l'ensemble de la population.

\section{b) Les "gestions nationales"}

Concernant cette seconde étape, l'étude fait ressortir également plusieurs facteurs explicatifs de la situation présente. Le premier facteur est le contexte politique dans lequel intervient cette prise en charge au niveau national. La disparition du système soviétique ouvre la voie à l'indépendance ukrainienne et à une transition institutionnelle vers un système démocratique. Dans ce contexte, la gestion post-accidentelle de l'accident de Tchernobyl devient un 
enjeu politique d'autant plus fort que la revendication à l'information et au débat public sur les conséquences de Tchernobyl se développe dans un contexte de censure et de secret (voir plus haut). La défense des intérêts des "victimes", la mise en place d'un système de compensation deviennent un thème politique majeur qui va porter au pouvoir une vague politique nouvelle. Elle débouchera sur le vote de la loi du 16 avril 1991. Succédant à une période de silence, le thème de Tchernobyl fait l'objet d'une surenchère et, notamment, vient incarner la revendication nationale à l'égard d'un système central affaibli. C'est ainsi que sont votés par le Parlement ukrainien des principes de compensation très généreux dont le pouvoir central soviétique est censé assurer le financement (ce projet se heurtera au refus des autorités soviétiques, suivi de la suspension par l'Etat ukrainien du règlement de sa contribution au budget central, etc., jusqu'à l'indépendance).

Dans ce contexte, il faut noter que le débat public qui se développe sur les conséquences de l'accident se focalise sur les effets de la phase B. Cette tendance est manifeste dans la loi ukrainienne du 16 avril 1991 dont une des caractéristiques est d'évaluer l'ensemble des effets de l'accident (phase A et phase B) à partir du niveau de contamination de la phase B. La loi détermine des zones de territoire contaminé (il y a 4 classes de zone) qui sont délimitées en fonction des évaluations de la dose-vie à recevoir par la population résidant sur ces zones, compte tenu de la contamination présente et future de leur territoire de résidence (phase $B$ ). L'ouverture de droits à compensation est liée au temps de résidence passé (phase $\mathrm{A}$ et $\mathrm{B}$ ) et futur (phase B) des personnes (liquidateurs, relogés, habitants des territoires contaminés) dans une zone et au classement de cette zone de résidence (de 1 à 4 ).

La loi ukrainienne du 16 avril 1991 repose, en outre, sur des concepts de gestion post-accidentelle qui sont, comme on l'a souligné plus haut, porteurs de nombreux effets pervers aux plans psychique et social.

\subsection{Modélisation des interactions}

Une modélisation des interactions entre les différents facteurs identifiés cidessus est proposée dans la figure 3 . On voit que ces interactions conduisent d'une part, à l'apparition d'effets différés de la phase $\mathrm{A}$ et, d'autre part, à l'attribution de ces effets à la phase $B$ par la population. Cette situation, qui résulte d'une combinaison perverse de ces différents facteurs, est funeste dans la mesure où elle conduit à une confusion entre les effets de la phase $\mathrm{A}$ et ceux de la phase $B$, avec de graves conséquences psychosociales.

Cette confusion revient à projeter une situation passée (comportant, semble-t-il, des risques non négligeables) dans le futur. Les effets différés de la phase $\mathrm{A}$ sont vécus comme des effets de la phase $\mathrm{B}$, c'est-à-dire comme les effets d'une contamination résiduelle qui ne pourra disparaître avant longtemps. Autant dire que la population ne voit aucune raison pour que ces effets disparaissent mais, au contraire, pense qu'ils doivent nécessairement augmenter, sentiment général dans la population que traduit bien cette phrase recueillie lors d'un entretien : "La catastrophe est un arbre qui pousse". 


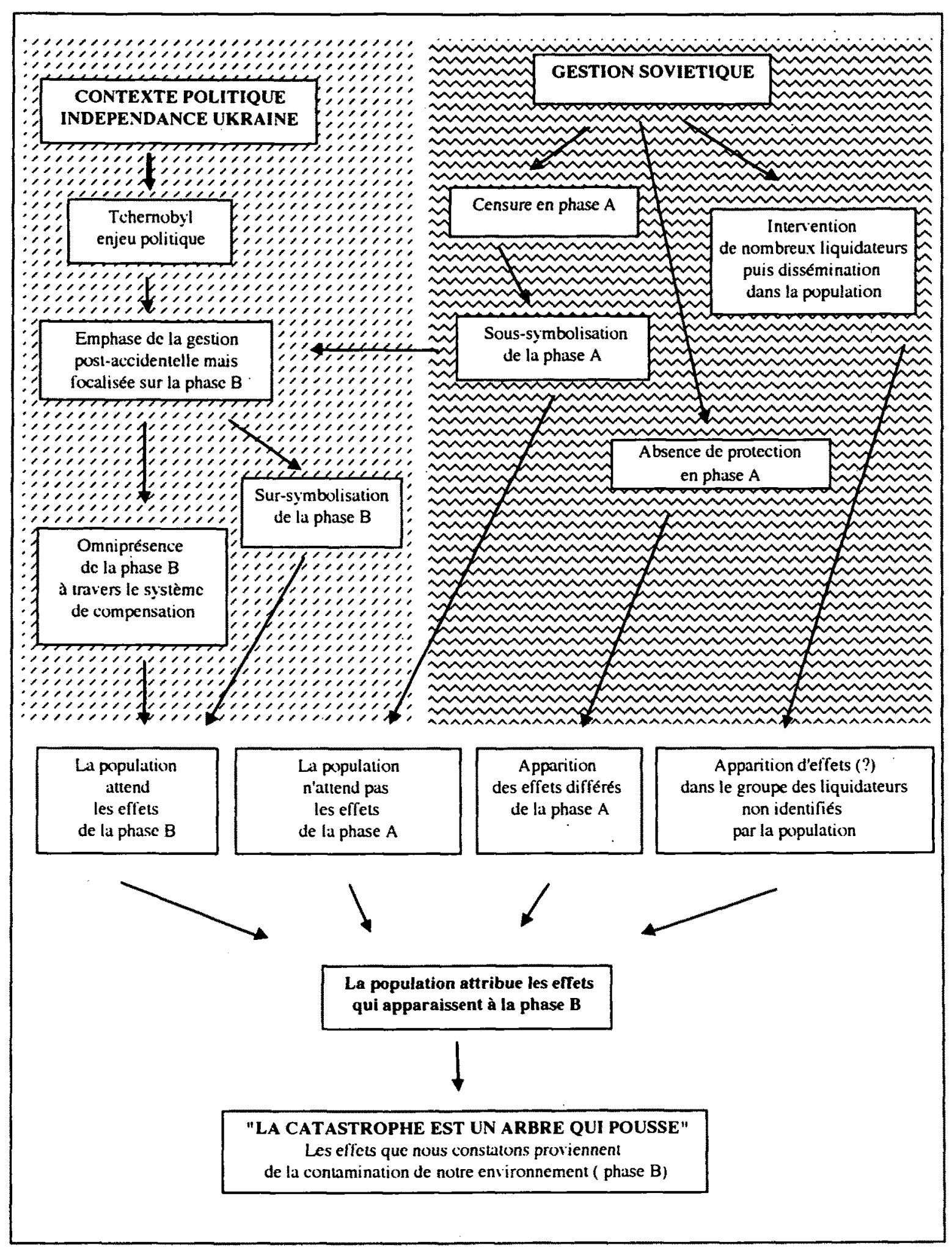

Fig. 3 - Eléments d'interprétation. Situation post-accidentelle de Tchernobyl en Ukraine. Some interpretation data on the Chernobyl post-accident situation in Ukraine. 


\section{Remerciements}

L'auteur remercie particulièrement Philippe Girard de l'Université de Caen avec qui il a réalisé les différentes enquêtes en Ukraine dont les conclusions sont citées dans cet article. Il remercie également Jacques Lochard du CEPN, Peter Allen du Robens Institute, Britt Marie Drott-Sjöberg du Center for risk research, et Neale Kelly de la Commission des Communautés Européennes (DGXII) pour leur contribution et leur soutien dans la réalisation de ce travail.

\section{RÉFÉRENCES}

[1] ALLEN P. - Social and psychological factors of Chernobyl aftermath. Guildford : Robens institute, University of Surrey, 1993.

[2] BARROIS C. - Les névroses traumatiques, Paris : Dunod, 1988.

[3] BATESON G. - Vers une écologie de l'esprit. Paris : Edition du Seuil, 1980, vol.2, 42-49.

[4] DROTT-SJÖBERG B.M. - Pilot study in Novozybkov, Russia. Stockholm : Center for risk research, Stockholm school of economics, 1992.

[5] GIRARD P., HÉRIARD DUBREUIL G. - Conséquences sociales et psychiques de l'accident de Tchernobyl en Ukraine, résultats des enquêtes menées en 1992 et 1993 , Paris : MUTADIS Consultants, 1994.

[6] GORBATCHEV M. - Discours du 14 mai 1986 et Revue de la presse soviétique concernant l'accident de Tchernobyl. In : Historical perspective of the countermeasures taken following the Chernobyl accident (CEPN report $\mathrm{N}^{\circ} 225$ ). Fontenay-auxRoses : CEPN, 1994, vol. 2, 311-317, 257-310.

[7] LEMOINE J.L. - La modélisation des systèmes complexes. Paris : Dunod, 1991.

[8] ORGANISATION MONDIALE DE LA SANTÉ (OMS). - Tchernobyl 7 ans après (Communication OMS/32 du 23 avril 1992). Genève : OMS, 1992.

[9] ORGANISATION MONDIALE DE LA SANTÉ (OMS). - Augmentation spectaculaire du caner de la thyroïde chez les enfants biélorusses et ukrainiens après l'accident de Tchernobyl (Communication OMS/84 du 29 oct. 1993). Genève : OMS, 1993.

[10] SIMON H. - Sciences des systèmes, sciences de l'artificiel. Paris : Dunod 1991.

[11] WILliamS D., PINCHERA A., KARAOGLOU A., CHADWICK K.H. - Thyroid cancer in children living near Chernobyl (Rapport EUR-15248 EN). Bruxelles : Commission des Communautés européennes, 1994. 